\title{
The Infinite Atom Dicke Maser Model II
}

\author{
E. B. Davies \\ Mathematical Institute, Oxford, U.K.
}

Received July 3, 1973

\begin{abstract}
We study the time evolution of a quantum field under a Hamiltonian constructed in an earlier paper by taking the limit as $n \rightarrow \infty$ of a Dicke maser model Hamiltonian for $n$ radiating atoms. We show that the radiation field converges to a dynamic equilibrium state independent of its initial state and that the strength of the field is inversely proportional to the square of the distance from the source. A number of variations of the Hamiltonian are also considered.
\end{abstract}

\section{Definition of the Hamiltonian}

In an earlier paper [2] we studied the limit as $n \rightarrow \infty$ of a sequence of Dicke maser model Hamiltonians $H_{n}$ on the spaces

$$
\left\{\otimes n \mathbb{C}^{2}\right\} \otimes \mathscr{F}
$$

where $\mathscr{F}$ is a Boson Fock space. The Hamiltonian $H_{n}$ describes a simple interaction between $n$ 2-level atoms and a quantum field with an infinite number of degrees of freedom. The limiting Hamiltonian $H$ was realised on

$$
l^{2}(\mathbb{Z}) \otimes \mathscr{F} \simeq L^{2}\{(-\pi, \pi), \mathscr{F}\} .
$$

In this paper we study the time evolution for the limiting Hamiltonian. This is done in substantially greater generality than is required for the development of [2]. The reason for this is that we wish to be able to treat a number of variations of the maser model - for example the case of multi-level atoms with a number of different emission modes.

We start by describing the quantum field in terms of a representation of the canonical commutation relations. We take a complex test function space $D$ dense in the single particle Hilbert space $D^{-} ; D$ is supposed to be a complete locally convex topological linear space under a topology stronger than the Hilbert space topology. The single particle Hamiltonian $S$ is supposed to be essentially self-adjoint on $D$ and the unitary group $e^{i S t}$ is supposed to leave $D$ invariant and to be jointly continuous from $\mathbb{R} \times D$ to $D$. The quantum field is defined on a Hilbert space $\mathscr{K}$ by a representation of the C.C.R.'s on $\mathscr{K}$. For each $f \in D$ there is a unitary 
operator $W(f)$ on $\mathscr{K}$ such that

$$
W(f) W(g)=W(f+g) \exp [i \operatorname{Im}\langle f, g\rangle / 2] .
$$

The self-adjoint field $\Phi(f)$ is then defined by

$$
W(f)=\exp [i \Phi(f)] .
$$

The representation is supposed to be cyclic with cyclic vector $\Omega \in \mathscr{K}$ and so is determined through the Gelfand-Segal construction by the functional

$$
E_{0}(f)=\langle W(f) \Omega, \Omega\rangle .
$$

We supposed that for all $f \in D$ and $t \in \mathbb{R}$

$$
E_{0}\left(e^{i S t} f\right)=E_{0}(f) .
$$

Then by $[4,8]$ there is a self-adjoint operator, the free Hamiltonian $H_{0}$, on $\mathscr{K}$ such that

and

$$
e^{i H_{0} t} \Omega=\Omega
$$

$$
e^{i H_{0} t} W(f) e^{-i H_{0} t}=W\left(e^{i S t} f\right)
$$

for all $f \in D$ and $t \in \mathbb{R}$. We remark that the above assumptions generalise [2] where we only considered the Fock space representation of the C.C.R.'s.

We describe the Hilbert space which is supposed to represent the collective behaviour of the infinite system of atoms. We let $X$ be a compact metric space with a specified probability measure $d x$. We also suppose that $G_{0}$ is a topological group acting jointly continuously on $X$ and leaving the probability measure invariant. $G_{0}$ represents the symmetries of the system including its time evolution, and has a unitary representation on the Hilbert space $L^{2}(X)$ defined by

$$
(g \varphi)(x)=\varphi(x g)
$$

for all $\varphi \in L^{2}(X)$. We suppose that we are given a finite-dimensional linear space $V$ of complex continuous functions on $X$ such that $V$ is invariant under $G_{0}$ and contains the function 1 . We let

$$
Y=\operatorname{Re}\left\{\sum_{r} f_{r} . \overline{f_{r}^{\prime}}: f_{r}, f_{r}^{\prime} \in V\right\}
$$

so that $Y$ is a finite dimensional linear space of real continuous function on $X$ invariant under the action of $G_{0}$. The above assumptions generalise the situation of [2] where $X=[0,2 \pi]$ and $G_{0}$ was the group of rotations of $X$. In [2] the subspace $V$ was not explicitly mentioned but it was in fact

$$
V=\left\{f: f(\theta): a+b e^{i \theta} \text { for some } a, b \in \mathbb{C}\right\} .
$$


The Hilbert space of the composite system is taken to be the space $L^{2}(X, \mathscr{K})$ of $\mathscr{K}$-valued square integrable functions on $X$. The time evolution of the system is defined by first introducing a certain infinitedimensional group of unitary operators on $L^{2}(X, \mathscr{K})$ as in [2].

Theorem 1.1. The formula

where

$$
\left(U_{h} \varphi\right)(x)=e^{i v(x)} W\left(\sum_{r} v_{r}(x) f_{r}\right) e^{-i H_{0} t}\{\varphi(x g)\}
$$

$$
h=\left\{y, \Sigma v_{r} f_{r}, t . g\right\} \in Y \times(V \otimes D) \times \mathbb{R} \times G_{0}
$$

and $\varphi \in L^{2}(X, \mathscr{K})$, defines a group $G$ of unitary operators on $L^{2}(X, \mathscr{K})$.

Proof. It is obvious that $U_{h}$ is a unitary operator. If $h, h^{\prime} \in G$ then

$$
\begin{aligned}
\left(U_{h} U_{h^{\prime}} \varphi\right)(x) & =e^{i y(x)} W\left(\sum_{r} v_{r}(x) f_{r}\right) e^{-i H_{0} t}\left\{\left(U_{h^{\prime}} \varphi\right)(x g)\right\} \\
= & e^{i y(x)} W\left\{\sum_{r} v_{r}(x) f_{r}\right\} e^{-i H_{0} t} e^{i y^{\prime}(x g)} W\left\{\sum_{s} v_{s}^{\prime}(x g) f_{s}^{\prime}\right\} \cdot e^{-i H_{0} t^{\prime}}\left\{\varphi\left(x g g^{\prime}\right)\right\} \\
= & e^{i y(x)+i\left(g y^{\prime}\right)(x)} W\left\{\sum_{r} v_{r}(x) f_{r}\right\} W\left\{e^{-i S t} \sum_{s}\left(g v_{s}^{\prime}\right)(x) f_{s}^{\prime}\right\} \cdot e^{-i H_{0}\left(t+t^{\prime}\right)}\left\{\varphi\left(x g g^{\prime}\right)\right\} \\
= & \exp \left[i y(x)+i\left(g y^{\prime}\right)(x)+i \operatorname{Im}\left\{\sum_{r s} v_{r}(x) \overline{\left(g v_{s}^{\prime}\right)(x)}\left\langle f_{r}, e^{-i S t} f_{s}^{\prime}\right\rangle\right\}\right] \\
& \cdot W\left\{\sum_{r} v_{r}(x) f_{r}+\sum_{s}\left(g v_{s}^{\prime}\right)(x)\left(e^{-i S t} f_{s}^{\prime}\right)\right\} \\
& \cdot e^{-i H_{0}\left(t+t^{\prime}\right)}\left\{\varphi\left(x g g^{\prime}\right)\right\}
\end{aligned}
$$

which is of the required form.

The expression for the multiplier (in the exp bracket) is very complicated, but it fortunately turns out not to be important. We point out that the group contains two subgroups corresponding to symmetries of the atomic system

$$
\left(U_{h} \varphi\right)(x)=e^{i v(x)} \varphi(x g)
$$

and symmetries of the quantum field

$$
\left(U_{h} \varphi\right)(x)=W\{f\} e^{-i H_{0} t} \varphi(x) .
$$

The total Hamiltonian of the system is defined as in [2] by selecting a one-parameter subgroup of $G$. We suppose that there is given a oneparameter subgroup of $G_{0}$, representing the time evolution of the system of atoms in the absence of any interaction with the field.

Theorem 1.2. The formula

$$
\left(V_{t} \varphi\right)(x)=e^{i \alpha(x, t)} W\{f(x, t)\} e^{-i H_{0} t}\{\varphi(x t)\}
$$


where $\varphi \in L^{2}(X, \mathscr{K})$ and $t \in \mathbb{R}$, defines a one-parameter unitary group on $L^{2}(X, \mathscr{K})$ if and only if $f, \alpha$ satisfy the cocycle equations

and

$$
f(x, s+t)=f(x, s)+e^{-i S s} f(x s, t)
$$

$\alpha(x, s+t)=\alpha(x, s)+\alpha(x s, t)+i \operatorname{Im}\left\langle f(x, s), e^{-i S s} f(x s, t)\right\rangle / 2 \bmod 2 \pi$.

All solutions of these equations satisfy

$$
f(x, 0)=0, \quad \alpha(x, 0)=0 .
$$

Proof. See [2].

The construction of all solutions of the cocycle equations seems a difficult problem but an important class of these are given in the following theorem.

Theorem 1.3. Suppose $g: X \rightarrow D$ and $\beta: X \rightarrow \mathbb{R}$ are continuous functions. Then the formulae

and

$$
f(x, s)=\int_{u=0}^{s} e^{-i S u} g(x u) d u
$$

$\alpha(x, s)=\int_{u=0}^{s} \beta(x u) d u+\frac{1}{2} \int_{u=0}^{s} \int_{1}^{u} \operatorname{Im}\left\langle e^{-i S t} g(x v), e^{-i S u} g(x u)\right\rangle d v d u($

define continuous cocycles with the properties

and

$$
\left.\frac{\partial}{\partial s} f(x, s)\right|_{s=0}=g(x)
$$

$$
-\left.\frac{\partial}{\partial s} \alpha(x, s)\right|_{s=0}=\beta(x) \text {. }
$$

Proof. See [2].

We are interested particularly in cocycles taking values in $V \otimes D$, and to describe these we need some further definitions. Since $V$ is invariant under the action of $G_{0}$ and hence of its one parameter subgroup $\mathbb{R}$, there is a finite orthonormal basis $v_{1}, \ldots, v_{n}$ of $V$ and constants $\omega_{1}, \ldots, \omega_{n} \in \mathbb{R}$ such that

$$
v_{r}(x t)=e^{i \omega_{r} t} v_{r}(x)
$$

for all $x \in X$ and $t \in \mathbb{R}$.

Theorem 1.4. If $g_{1}, \ldots, g_{n} \in D$ then the equations

$$
f(x, t)=\sum_{r=1}^{n} v_{r}(x) \int_{u=0}^{t} e^{-i\left(S-\omega_{r}\right) u} g_{r} d u
$$


and

$\alpha(x, t)=\frac{1}{2} \sum_{r, s} \operatorname{Im}\left\{v_{r}(x) \overline{v_{s}(x)} \cdot \int_{u=0}^{t} \int_{i=0}^{u} e^{i\left(\omega_{r} v-\omega_{s} u\right)}\left\langle e^{-i S v} g_{r}, e^{-i S u} g_{s}\right\rangle d v d u\right\}$

define continuous cocycles.

Proof. This is obtained by substituting into Theorem 1.3 the choices $\beta=0$ and

$$
g(x)=\sum_{r=1}^{n} v_{r}(x) g_{r} .
$$

To summarise, we have shown that given $g_{1}, \ldots, g_{n} \in D$ there is a strongly continuous one parameter unitary group $V_{t}$ defined on $L^{2}(X, \mathscr{K})$ by Eqs. (1.17), (1.26) and (1.27). The Hamiltonian $H$ is then defined as the self-adjoint operator such that $V_{t}=e^{-i H t}$ in the usual sense. It may be shown that the Hamiltonian is given explicitly by

$(H \varphi)(x)=H_{0}\{\varphi(x)\}-\sum_{r=1}^{n} \Phi\left\{v_{r}(x) g_{r}\right\}\{\varphi(x)\}+\left.i \frac{\partial}{\partial t} \varphi(x t)\right|_{t=0}$.

This formal equation may be given a precise sense as in [2], but we shall not do this as we shall be working with the unitary group $V_{t}$, which is already rigorously defined.

\section{Convergence to Equilibrium of the Field}

We now move on to a description of the evolution of the field for the Hamiltonian $H$ defined in the last section, and in particular the behaviour in the limit $t \rightarrow \infty$. There are three conditions of great importance which we shall use.

(i) For all $f, g \in D$

$$
\lim _{t \rightarrow \infty}\left\langle e^{-i S t} f, g\right\rangle=0 .
$$

Moreover for all $r=1, \ldots, n$

$$
\lim _{t \rightarrow \infty} \int_{0}^{t}\left\langle e^{-i\left(S-\omega_{r}\right) u} f, g\right\rangle d u
$$

exists and is finite.

(ii) The representation $W$ has the asymptotic product decomposition property; in other words for all $f, g \in D$

$$
\lim _{t \rightarrow \infty} E_{0}\left(f+e^{-i S t} g\right)=E_{0}(f) E_{0}(g) .
$$

(iii) The action of $\mathbb{R}$ on $X$ is ergodic. 
Theorem 2.1. Suppose conditions (i) and (ii) are satisfied. If $\psi_{1}, \psi_{2} \in L^{2}(X, \mathscr{K})$ are invariant under the action of $\mathbb{R}$ on $X$ then

where

$$
\begin{gathered}
\lim _{t \rightarrow \infty}\left\langle W(f) e^{-i H t} \psi_{1}, e^{-i H t} \psi_{2}\right\rangle \\
=E_{0}(f)\left\langle\psi_{1}, \psi_{2}\right\rangle \int_{X} \exp \left[-i \operatorname{Im}\left(\sum_{r=1}^{n} v_{r}(x) \varphi_{r}(f)\right)\right] d x
\end{gathered}
$$

Proof. By the linearity with respect to $\psi$ and $\psi^{\prime}$ and the assumption that $\Omega$ is a cyclic vector for the representation of the C.C.R.'s we only need to prove this for the case

$$
\psi_{i}(x)=\lambda_{i}(x) W\left(g_{i}\right) \Omega
$$

where $\lambda_{1}, \lambda_{2}$ are arbitrary continuous functions on $X$ and $g_{1}, g_{2}$ are arbitrary elements of $D$. With these choices

$$
\begin{gathered}
\left\langle W(f) e^{i H t} \psi_{1}, e^{-\imath H t} \psi_{2}\right\rangle \\
=\int_{X}\left\langle W(f) e^{i \alpha(x, t)} W\{f(x, t)\} e^{-i H_{0} t} \lambda_{1}(x t) W\left(g_{1}\right) \Omega,\right. \\
\left.\quad e^{i x(x, t)} W\{f(x, t)\} e^{-i H_{0} t} \lambda_{2}(x t) W\left(g_{2}\right) \Omega\right\rangle d x \\
=\int_{X} \lambda_{1}(x t) \overline{\lambda_{2}(x t)}\left\langle W(f) W\{f(x, t)\} W\left\{e^{-i S t} g_{1}\right\} \Omega,\right. \\
\left.W\{f(x, t)\} W\left\{e^{-i S t} g_{2}\right\} \Omega\right\rangle d x \\
=F(t) \int_{X} \lambda_{1}(x t) \overline{\lambda_{2}(x t)} \exp [i \operatorname{Im}\langle f, f(x, t)\rangle] d x
\end{gathered}
$$

where

$$
\begin{aligned}
F(t)= & \left\langle W(f) W\left\{e^{-i S t} g_{1}\right\} \Omega, W\left\{e^{-i S t} g_{2}\right\} \Omega\right\rangle \\
= & \exp \left[-i \operatorname{Im}\left\langle e^{-i S t} g_{2}, f+e^{-i S t} g_{1}\right\rangle / 2\right. \\
& \left.+i \operatorname{Im}\left\langle f, e^{-i S t} g_{1}\right\rangle / 2\right] \cdot E_{0}\left(f+e^{-i S t} g_{1}-e^{-i S t} g_{2}\right) \\
= & \exp \left[-i \operatorname{Im}\left\langle g_{2}, g_{1}\right\rangle / 2\right] E_{0}(f) E_{0}\left(g_{1}-g_{2}\right)+o(1)
\end{aligned}
$$

as $t \rightarrow \infty$, by conditions (i) and (ii).

Therefore

$$
=E_{0}(f)\left\langle W\left(g_{1}\right) \Omega, W\left(g_{2}\right) \Omega\right\rangle+o(1) .
$$

$$
\begin{aligned}
\left\langle W(f) e^{-i H t} \psi_{1}, e^{-t H t} \psi_{2}\right\rangle \\
=E_{0}(f)\left\langle W\left(g_{1}\right) \Omega, W\left(g_{2}\right) \Omega\right\rangle \int_{X} \lambda_{1}(x t) \overline{\lambda_{2}(x t)} \\
\quad \cdot \exp [i \operatorname{Im}\langle f, f(x, t)\rangle] d x+o(1) \\
=E_{0}(f) \int_{X}\left\langle\psi_{1}(x t), \psi_{2}(x t)\right\rangle \exp [i \operatorname{Im}\langle f, f(x, t)\rangle] d x+o(1) \\
=E_{0}(f) \int_{X}\left\langle\psi_{1}(x), \psi_{2}(x)\right\rangle \exp [i \operatorname{Im}\langle f, f(x, t)\rangle] d x+o(1)
\end{aligned}
$$


by the invariance of $\psi_{1}$ and $\psi_{2}$,

where

$$
=E_{0}(f) \int_{X}\left\langle\psi_{1}(x), \psi_{2}(x)\right\rangle \exp [i G(x)] d x+o(1)
$$

$$
\begin{aligned}
G(x) & =\lim _{t \rightarrow \infty} \operatorname{Im}\langle f, f(x, t)\rangle \\
& =\operatorname{Im}\left\{\sum_{r=1} v_{r}(x) \lim _{t \rightarrow \infty}\left\langle f, \int_{0}^{t} e^{-i\left(S-\omega_{r}\right) u} g_{r} d u\right\rangle\right\} \\
& =-\operatorname{Im}\left\{\sum_{r=1}^{n} v_{r}(x) \varphi_{r}(f)\right\} .
\end{aligned}
$$

The above theorem is inadequate in as much as it only applies to invariant states $\psi_{1}$ and $\psi_{2}$. In the following theorem we use the notation

$$
\operatorname{Lim}_{t \rightarrow \infty} k(t)=\lim _{t \rightarrow \infty} t^{-1} \int_{0}^{t} k(u) d u .
$$

noting that if the ordinary limit exists, then so does the generalised limit, and both are equal.

Theorem 2.2. Suppose conditions (i), (ii) and (iii) are satisfied. Then for any $\psi_{1}, \varphi_{2} \in L^{2}(X, \mathscr{K})$

$$
\begin{gathered}
\operatorname{Lim}_{t \rightarrow \infty}\left\langle W(f) e^{-\imath H t} \psi_{1}, e^{-i H t} \psi_{2}\right\rangle \\
=E_{0}(f)\left\langle\psi_{1}, \psi_{2}\right\rangle \int_{X} \exp \left[-i \operatorname{Im}\left\{\sum_{r=1}^{n} v_{r}(x) \varphi_{r}(f)\right\}\right] d x .
\end{gathered}
$$

Proof. From Eq. (2.9) we get for large $t$

Therefore

$$
\begin{gathered}
\left\langle W(f) e^{-i H t} \psi_{1}, e^{-i H t} \psi_{2}\right\rangle \\
=E_{0}(f) \int_{X}\left\langle\psi_{1}(x t), \psi_{2}(x t)\right\rangle \exp [i G(x)] d x+o(1) .
\end{gathered}
$$

$$
\begin{aligned}
\operatorname{Lim}_{t \rightarrow \infty} & \left\langle W(f) e^{-i H t} \psi_{1}, e^{-i H t} \psi_{2}\right\rangle \\
& =E_{0}(f) \lim _{t \rightarrow \infty} t^{-1} \int_{0}^{t} \int_{X}\left\langle\psi_{1}(x s), \psi_{2}(x s)\right\rangle \exp [i G(x)] d x d s \\
& =E_{0}(f) \int_{X}\left\langle\psi_{1}(x), \psi_{2}(x)\right\rangle d x \int_{X} \exp [i G(x)] d x
\end{aligned}
$$

by condition (iii) - see [6]. This concludes the proof.

Theorem 2.3. Suppose conditions (i), (ii), and (iii) are satisfied. Then for any positive operator $\varrho$ on $L^{2}(X, \mathscr{K})$ such that $\operatorname{tr}[\varrho]=1$.

$$
\begin{gathered}
\operatorname{Lim}_{t \rightarrow \infty} \operatorname{tr}\left[W(f) e^{-\imath t H} \varrho e^{i t H}\right] \\
=E_{0}(f) \int_{X} \exp \left[-i \operatorname{Im}\left\{\sum_{r=1}^{n} v_{r}(x) \varphi_{r}(f)\right\}\right] d x .
\end{gathered}
$$


Proof. For operators $\varrho$ of finite rank this is obtained from Theorem 2.2 by linearity. For general $\varrho$ we then use simple density arguments.

This is the central theorem of the paper. Physically it states that the quantum field converges, in the mean, to a state independent of its initial state. We continue this section by studying cases where the right hand side of Eq. (2.16) may be simplified.

Theorem 2.4. Suppose that condition (iii) holds and that $\omega_{1}, \ldots, \omega_{n}$ are rationally independent. Then

$$
\int_{X} \exp \left[-i \operatorname{Im}\left\{\sum_{r=1}^{n} v_{r}(x) \varphi_{r}(f)\right\}\right] d x=\prod_{r=1}^{n} J_{0}\left(\left|\varphi_{r}(f)\right|\right) .
$$

Proof. We take from [6] the following facts. The action of $\mathbb{R}$ on $X$ defines a unitary group on $L^{2}(X)$. The eigenvalues form a countable subgroup of $\mathbb{R}$ and each eigenvalue is of multiplicity one, each eigenfunction being of absolute value one almost everywhere. A set $v_{1}, \ldots, v_{n}$ of eigenfunctions are probabilistically independent if and only if the corresponding eigenvalues are rationally independent. If this occurs then

$$
\begin{aligned}
I & \equiv \int_{X} \exp \left[-i \operatorname{Im}\left\{\sum_{r=1}^{n} v_{r}(x) \varphi_{r}(f)\right\}\right] d x \\
& =\prod_{r=1}^{n} \int_{X} \exp \left[-i \operatorname{Im}\left\{v_{r}(x) \varphi_{r}(f)\right\}\right] d x .
\end{aligned}
$$

We next observe that for any function $h$

Therefore

$$
\begin{aligned}
\int_{X} h\left\{v_{r}(x)\right\} d x=\frac{1}{2 \pi} \int_{0}^{2 \pi} \int_{X} h\left\{v_{r}\left(x \cdot \theta \omega_{r}^{-1}\right)\right\} d x d \theta \\
=\frac{1}{2 \pi} \int_{X} \int_{0}^{2 \pi} h\left\{e^{i \theta} v_{r}(x)\right\} d \theta d x \\
=\frac{1}{2 \pi} \int_{0}^{2 \pi} h\left\{e^{i \theta}\right\} d \theta .
\end{aligned}
$$

$$
\begin{aligned}
I & =\prod_{r=1}^{n}\left[\frac{1}{2 \pi} \int_{0}^{2 \pi} \exp \left[-i \operatorname{Im}\left\{e^{i \theta} \varphi_{r}(f)\right\}\right] d \theta\right] \\
& =\prod_{r=1}^{n} J_{0}\left(\left|\varphi_{r}(f)\right|\right) .
\end{aligned}
$$

The case where two or more of the frequencies are rationally related seems not to be capable of such simple calculation. Such a solution would arise in the description of a coherent source which radiated harmonics of a given frequency, with phase relation-ships between the harmonics. 
There is another case where the integral may be simply calculated, which we start by motivating. The Dicke maser model was treated in [2] with the Hamiltonian

$$
H=\omega J_{3}+H_{0}+\lambda\left\{J_{-} a^{*}(b)+J_{+} a(b)\right\}
$$

which commutes with the number operator. However, one also frequently considers the more general Hamiltonian $[3,5]$

$H=\omega J_{3}+H_{0}+\lambda\left\{J_{-} a^{*}\left(b_{1}\right)+J_{+} a\left(b_{1}\right)+J_{-} a\left(b_{2}\right)+J_{+} a^{*}\left(b_{2}\right)\right\}$.

If one recalculates [2] with this more general Hamiltonian, then one finds a limit of much the same form, except that in the cocycle $f(\theta, t)$ one has two terms corresponding to both frequencies $\pm \omega$. The integral of Eq. (2.16) may be simplified using the following theorem.

Theorem 2.5. Suppose that condition (iii) holds and

$$
v_{ \pm}(x t)=e^{ \pm i \omega t} v_{ \pm}(x)
$$

for all $x \in X$ and $t \in \mathbb{R}$. Then

$$
\begin{gathered}
\int_{X} \exp \left[-i \operatorname{Im}\left\{v_{+}(x) \varphi_{+}(f)+v_{-}(x) \varphi_{-}(f)\right\}\right] d x \\
=J_{0}\left(\left|\varphi_{+}(f)-\overline{\varphi_{-}(f)}\right|\right) .
\end{gathered}
$$

Proof. By ergodicity [6] we know that $v_{-}(x)=\overline{v_{+}(x)}$ for all $x \in X$. Therefore the left hand side of Eq. (2.24) is equal to

$$
\int_{X} \exp \left[-i \operatorname{Im} v_{+}(x)\left\{\varphi_{+}(f)-\overline{\varphi_{-}(f)}\right\}\right] d x=J_{0}\left(\left|\varphi_{+}(f)-\overline{\varphi_{-}(f)}\right|\right)
$$

as before.

\section{Properties of the Equilibrium State of the Field}

The next problem consists of an analysis of the final state of the field. We concentrate attention for the sake of simplicity on the case where the initial representation of the C.C.R.'s is the Fock space representation, and where there is only one radiation mode involved. We have shown in the last section that the field evolves from its initial state to a final dynamic equilibrium state with expectation function

where

$$
E(f)=\exp \left[-\|f\|^{2} / 4\right] \cdot J_{0}(|\varphi(f)|)
$$

$$
\varphi(f)=\lim _{t \rightarrow \infty} \int_{0}^{t}\left\langle e^{-i(S-\omega) u} g, f\right\rangle d u .
$$

The expectation function $E$ defines a new representation of the C.C.R.'s [1], which in general will be inequivalent to the Fock representation, since the linear functional $\varphi$ on $D$ will be unbounded. 
We take the particular case where $D$ is the Schwarz space in $L^{2}\left(\mathbb{R}^{3}\right), S$ is the operator $-\Delta / 2$, which is essentially self adjoint on $D$, and the constant $\omega$ is non-negative (the more normal case). It can be readily verified that condition (i) of the last section is satisfied.

Theorem 3.1. The functional $\varphi$ is given on $D$ by

where

$$
\varphi(f)=\int_{\mathbb{R}^{3}} \varphi(x) \overline{f(x)} d^{3} x
$$

$$
\varphi(x)=-i \int_{\mathbb{R}^{3}} g(y) \frac{e^{i \sqrt{2 \omega}\|x-y\|}}{2 \pi\|x-y\|} d^{3} y
$$

is a bounded continuous function on $\mathbb{R}^{3}$.

Proof. We have

$$
\begin{aligned}
\varphi(f) & =\lim _{\varepsilon \downarrow 0} \int_{0}^{\infty} e^{-\varepsilon u}\left\langle e^{-i(S-\omega) u} g, f\right\rangle d u \\
& =-i \lim _{\varepsilon \downarrow 0}\left\langle(S-\omega-i \varepsilon)^{-1} g, f\right\rangle \\
& =-i \lim _{\varepsilon \downarrow 0} \int_{\mathbb{R}^{3}} \frac{\hat{g}(y) \overline{\hat{f}(y)} d^{3} y}{\frac{1}{2}\|y\|^{2}-\omega-i \varepsilon} .
\end{aligned}
$$

Now it is well known that if $\lambda$ is not a negative real number

Therefore

$$
\int_{\mathbb{R}^{3}} \frac{e^{-\sqrt{\lambda}\|x\|}}{4 \pi\|x\|} e^{i\langle x, y\rangle} d^{3} x=\left(\lambda+\|y\|^{2}\right)^{-1} .
$$

$$
\begin{aligned}
\varphi(f) & =-2 i \lim _{\varepsilon \downarrow 0} \iint g(y) \overline{f(x)} \frac{e^{i \sqrt{2(\omega+i \varepsilon)}\|x-y\|}}{4 \pi\|x-y\|} d^{3} x d^{3} y \\
& =-i \iint g(y) \overline{f(x)} \frac{e^{i \sqrt{2 \omega}\|x-y\|}}{2 \pi\|x-y\|} d^{3} x d^{3} y \\
& =\int_{\mathbb{R}^{3}} \varphi(x) \overline{f(x)} d^{3} x .
\end{aligned}
$$

The stated properties of $\varphi$ follows from simple estimates [7].

Theorem 3.2. The expectation function $E$ is locally Fock. That is, for each bounded region $U$ in $\mathbb{R}^{3}$ there is a density matrix $\varrho_{U}$ on the Fock space $\mathscr{F}_{U}$ over $L^{2}(U)$ such that

$$
E(f)=\operatorname{tr}\left[\varrho_{U} W(f)\right]
$$

for all $f \in D$ with support in $U$. The expected number of particles in the region $U$ is finite and is given by

$$
\operatorname{tr}\left[\varrho_{U} N_{U}\right]=\frac{1}{2} \int_{U}|\varphi(x)|^{2} d^{3} x .
$$


Proof. We follow the methods of [1], the reason for the validity of the theorem being that restricted to $L^{2}(U), \varphi$ is a bounded functional. Let us define

and define

$$
\varphi_{U}(x)=\left\{\begin{array}{lll}
\varphi(x) & \text { if } & x \in U \\
0 & \text { otherwise }
\end{array}\right.
$$

$$
\varrho_{U}=\frac{1}{2 \pi} \int_{0}^{2 \pi} W\left\{e^{i \theta} \varphi_{U}\right\} \Omega \otimes W\left\{e^{i \theta} \varphi_{U}\right\} \Omega^{-} d \theta
$$

where $\Omega$ is the Fock vacuum. Then $\varrho_{U}$ is a density matrix on $\mathscr{F}_{U}$ and if $f \in D$ has support in $U$

$$
\begin{aligned}
\operatorname{tr} & {\left[\varrho_{U} W(f)\right]=\frac{1}{2 \pi}-\int_{0}^{2 \pi}\left\langle W(f) W\left\{e^{i \theta} \varphi_{U}\right\} \Omega, W\left\{e^{i \theta} \varphi_{U}\right\} \Omega\right\rangle d \theta } \\
& =\frac{1}{2 \pi} \int_{0}^{2 \pi} \exp \left[i \operatorname{Im}\left\langle f, e^{i \theta} \varphi_{U}\right\rangle\right]\langle W(f) \Omega, \Omega\rangle d \theta \\
& =\frac{1}{2 \pi} \exp \left[-\|f\|^{2} / 4\right] \int_{0}^{2 \pi} \exp \left[i \operatorname{Im}\left\{e^{-i \theta}\left\langle f, \varphi_{U}\right\rangle\right\}\right] d \theta \\
& =\exp \left[-\|f\|^{2} / 4\right] \cdot J_{0}\left(\left|\left\langle\varphi_{U}, f\right\rangle\right|\right) \\
& =\exp \left[-\|f\|^{2} / 4\right] J_{0}(|\varphi(f)|) \\
& =E(f)
\end{aligned}
$$

as required. The expected number of particles in the region is given by

$$
\begin{aligned}
\operatorname{tr}\left[\varrho_{U} N_{U}\right] & =\frac{1}{2 \pi} \int_{0}^{2 \pi}\left\langle N W\left\{e^{i \theta} \varphi_{U}\right\} \Omega, W\left\{e^{i \theta} \varphi_{U}\right\} \Omega\right\rangle d \theta \\
& =\frac{1}{2}\left\|\varphi_{U}\right\|^{2} \\
& =\frac{1}{2} \int_{U}|\varphi(x)|^{2} d^{3} x .
\end{aligned}
$$

We have shown that the particles in the field are distributed throughout space with the density

$$
\varrho(x)=\frac{1}{2}|\varphi(x)|^{2} .
$$

Physically this is the equilibrium intensity of radiation due to the finite source, which may be considered to be located within the support set of the function $g$. The main interest centres on the form of the function $\varrho$ a long distance from the origin compared with the wavelength of the emitted radiation. The following theorem, a very simple case of one in [7], states that the intensity of radiation varies with the inverse square of the distance from the source. 
Theorem 3.3. Suppose that $g$ is a continuous function of compact support and that $u$ is a unit vector in $\mathbb{R}^{3}$. Then

$$
\lim _{r \rightarrow \infty} r^{2} \varrho(r u)=\pi|\hat{g}(\sqrt{2 \omega} u)|^{2} .
$$

Proof. For large $r>0$

$$
\begin{aligned}
r \varphi(r u) & =-i \int_{\mathbb{R}^{3}} r g(y) \frac{e^{i \sqrt{2 \omega \|}\|r u-y\|}}{2 \pi\|r u-y\|} d^{3} y \\
& \sim-\frac{i}{2 \pi} \int_{\mathbb{R}^{3}} g(y) e^{i \sqrt{2} \bar{\omega}(r-\langle u, y\rangle)} d^{3} y \\
& =\sqrt{2 \pi} e^{i \sqrt{2 \omega} r} \hat{g}(\sqrt{2 \omega} u) .
\end{aligned}
$$

We next turn to the situation described at the end of Section 2, where the Hamiltonian has both position and negative energy terms of the same frequency.

Theorem 3.4. If the quantum field is in the state

$$
E(f)=\exp \left[-\|f\|^{2} / 4\right] \cdot J_{0}\left(\left|\varphi_{+}(f)-\overline{\varphi_{-}(f)}\right|\right)
$$

then the particle density function $\varrho$ satisfies

$$
\lim _{r \rightarrow \infty} r^{2} \varrho(r u)=\pi\left|\hat{g}_{+}(\sqrt{2 \pi} u)\right|^{2}
$$

for every unit vector $u$ in $\mathbb{R}^{3}$.

Proof. We have to estimate

where

$$
\begin{gathered}
\varphi_{+}(f)-\overline{\varphi_{-}(f)} \\
=\int_{\mathbb{R}^{3}}\left\{\varphi_{+}(x) \overline{f(x)}-\overline{\varphi_{-}(x)} f(x)\right\} d^{3} x
\end{gathered}
$$

$$
\varphi_{+}(x)=-i \int_{\mathbb{R}^{3}} g_{+}(y) \frac{e^{i \sqrt{2 \omega}\|x-v\|}}{2 \pi\|x-y\|} d^{3} y
$$

has already been estimated. On the other hand

$$
\begin{aligned}
\varphi_{-}(f) & =\lim _{t \rightarrow \infty} \int_{0}^{t}\left\langle e^{-i(S+(0) u} g_{-}, f\right\rangle d u \\
& =-i\left\langle(S+\omega)^{-1} g_{-}, f\right\rangle \\
& =-i \int_{\mathbb{R}^{3}} \frac{\hat{g}_{-}(y) \overline{\hat{f}(y)} d^{3} y}{\frac{1}{2}\|y\|^{2}+\omega} \\
& =\int_{\mathbb{R}^{3}} \varphi_{-}(x) \overline{f(x)} d^{3} x
\end{aligned}
$$

where

$$
\varphi_{-}(x)=-i \int_{\mathbb{R}^{3}} g_{-}(y) \frac{e^{-\sqrt{2 \omega}\|x-y\|}}{2 \pi\|x-y\|} d^{3} y \text {. }
$$


It is clear that for large $r$

$$
\varphi_{-}(r u)=0\left(e^{-\sqrt{2 \omega r} r} / r\right)
$$

which is negligible compared with $\varphi_{+}(r u)$.

The negative frequency term does therefore contribute to the final equilibrium state of the field, but since $\varphi_{-}$is a bounded linear functional on $\mathbb{R}^{3}$ it contributes only a finite number of particles which remain in the neighbourhood of the source.

\section{References}

1. Araki,H., Woods, E.J.: Representations of the C.C.R.'s for a non-relativistic infinite free Bose gas. J. Math. Phys. 4, 637-662 (1963)

2. Davies, E. B.: Exact dynamics of an infinite-atom Dicke maser model. Commun. math. Phys. 33, 187--205 (1973)

3. Dicke, R.H.: Coherence in spontaneous radiation processes. Phys. Rev. 93, 99--110 (1954)

4. Emch, G.G.: Algebraic methods in statistical mechanics and quantum field theory. New York: J. Wiley and Sons. 1972

5. Fain, V.M., Khanin, Ya I.: Quantum electronics, Vol. 1. Oxford: Pergamon Press 1969

6. Halmos, P. R.: Ergodic theory. New York: Chelsea Publ. Co. 1956

7. Ikebe, T.: Eigenfunction expansions associated with the Schrödinger operators and their applications to scattering theory. Arch. Rat. Mech. Anal. 5, 1-34 (1960)

8. Segal, I. E.: Foundations of the theory of dynamical systems of infinitely many degrees of freedom I. Mat.-Fys. Medd. Danske Vid. Selsk. 31 (12), 39 (1959)

E. B. Davies

Mathematical Institute

24-29 St. Giles

Oxford, England 
